\title{
Evaluation OF Mimo System CAPACITY OVER RAYLEIGH FADING CHANNEL
}

\author{
Emad. Mohamed and A.M.Abdulsattar \\ Alhdba University College, Mousel, Iraq
}

\begin{abstract}
High transmission data rate, spectral efficiency and reliability are essential for future wireless communications systems. MIMO (multi-input multi-output) diversity technique is a band width efficient system achieving high data transmission which eventually establishing a high capacity communication system. Without needing to increase the transmitted power or the channel bandwidth, gain in capacity can be considerably improved by varying the number of antennas on both sides. Correlated and uncorrelated channels MIMO system was considered in this paper for different number of antennas and different SNR over Rayleigh fading channel. At the transmitter both CSI(channel state information) technique and Water filling power allocation principle was also considered in this paper.
\end{abstract}

\section{KEYWORDS}

Capacity, MIMO, CSI, Rayleigh fading, Water Filling.

\section{INTRODUCTION}

Limits of Channels capacity becomes an important criteria in modern communication system due to large demand on wireless communication services. In the late 1940s, Claude Shannon pioneered the channel capacity by developing a mathematical theory of communication based on the notation of mutual information between the input and output of the transmission channel [1].The earlier publication in the wireless communications field was considered the capacity of SISO (single-input single-output), SIMO (single-input multiple-output)and MISO (multiple-input single-output)over Rayleigh fading channels [2]. SIMO and MISO represents a kind of receiving and transmitting diversity respectively, where the system capacity logarithmic growth (bounded) versus number of antennas. During the past few years, Multiple-input multiple-output (MIMO) has becomes an important subject of research in wireless activities[1].Without increasing the channel bandwidth or transmitted power, MIMO channel capacity can be efficiently improved, by adding antenna elements at both transmitter and receiver[3]. This growing in channel capacity becomes more realistic in the case where there is enough multipath channels, that is, a rich scattering environment. The main idea behind MIMO system, is its ability to turn multipath phenomena, traditionally a pitfall of wireless transmission, into a benefit for increasing system capacity which becomes a promising solution for high bit rate wireless services [4].The correlation phenomena that appears in MIMO channel is one of the parameters that strongly affect the system performance. Correlation decreases with an increase in the distance between antennas, this distance must be not less than $\lambda / 2$, where $\lambda$ is the signal wave length. Correlation increasing leads to decreasing in system capacity [5].CSI technique was introduced in MIMO system for a goal to improve system capacity. The main idea behind CSI technique is to estimate the channel properties at the transmitter site, which gives a good evaluation for fading and scattering happened in the communication link. The rest of this paper is organized as follows. 
Channel capacity for SISO and different antenna diversity using, SIMO, MISO and MIMO techniques was presented in section 2. CSI and Equal power allocating criteria was presented in Sections 3 and 4 respectively. Section 5 provides description of correlated MIMO channel. Ergodic capacity is introduced in section 6. Finally, a MATLAB simulation results was given in Section 7.

\section{Channel Capacity}

A communication channel is a medium that is used to transmit signals from a transmitter to a receiver. During a transmission, the signals at the receiver may be disturbed by noise along with channel distortions. However, the noise and channel distortions can be differentiated because the channel distortions are a fixed function applied to the signals while the noise has statistical and unpredictable perturbations. Let Considering firstly a discrete-time additive white Gaussian noise (AWGN) channel shown in Figure.1, with a channel input/output relationship represented by (1).

$$
y[t]=x[t]+N[t]
$$

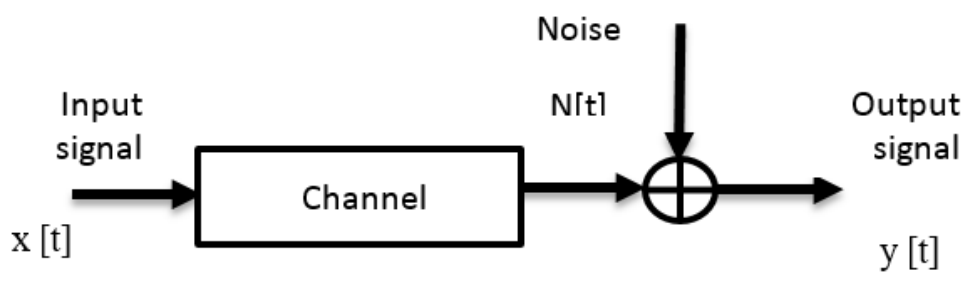

Figure 1. Discrete-time AWGN channel

Assume that it is possible to reliably distinguish $M$ different signal states in a period time of duration $T$ over a communication channel. Shannon [6-8] showed that if $T \rightarrow \infty$ the rate of transmission $R$ approaches the channel capacity $C$ in terms of the number of bits per transmission.The information (channel) capacity of a Gaussian channel is then represented by(2).

$$
\begin{aligned}
R & \leq C=\operatorname{Lim}_{T \rightarrow \infty}\left(\frac{\log _{2} M}{T}\right) \\
& =\frac{1}{2} \log _{2}\left(1+\frac{P}{N}\right)
\end{aligned}
$$

Where, $\frac{P}{N}$ is the signal to noise ratio.

\subsection{Siso CAPACITY}

For SISO system shown in the Figure2, the capacity is represented by equation (3) [9].
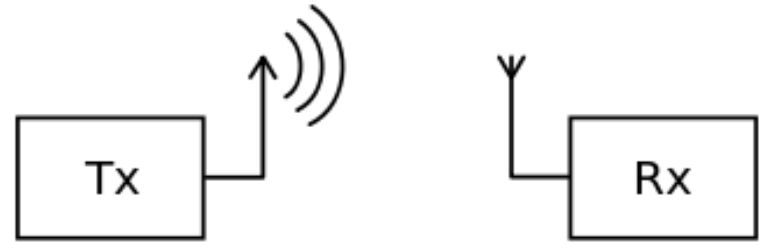

Figure 2.SISO channel 
International Journal of Computer Science \& Engineering Survey (IJCSES) Vol.6, No.3, June 2015

$$
C=\log _{2}\left(1+\rho|h|^{2}\right) \quad \text { bits } / \mathrm{s} / \mathrm{Hz}
$$

Where, $h$ is the normalized complex gain of a fixed wireless channel, $\rho$ is the SNR at the received antenna.

\subsection{Simo CAPACITY}

A block diagram of SIMO channel is shown in the Figure (3).
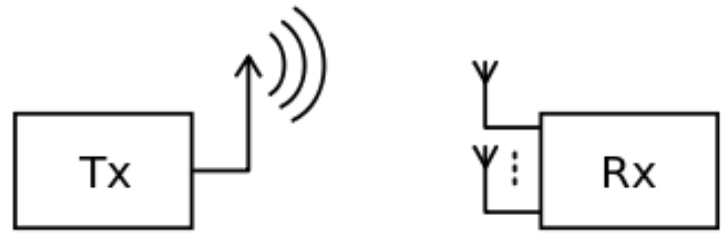

Figure 3.SIMO channel

SIMO channel mathematical model can be rewritten as:

$$
\mathbf{y}=\mathbf{h} x+\mathrm{N}
$$

Where $\mathbf{h}$ is the $n_{r} x$ lchannel matrix represented by(5).

$$
\mathbf{h}=\left[h_{1}, h_{2}, \ldots \ldots, h_{n r}\right]^{T}
$$

Where the elements $h i, i=1,2, \ldots \ldots, n_{r}$ represents the channel gain between the single transmitter antenna and the $i_{\text {th }}$ receiver antenna over a symbol period, and $n_{r}$ is the received antennas. A discrete-time SIMO channel capacity is represented by (6) [9]:

\subsection{Miso CAPACITY}

MISO block diagram is illustrated in the Figure (4).
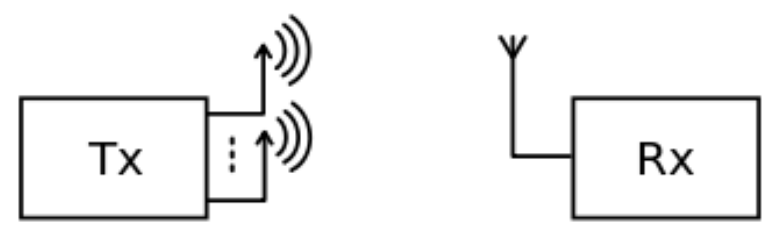

Figure 4.MISO channel

MISO channel mathematical model can be rewritten as:

$$
y=\mathbf{h} \mathbf{x}+N
$$


Where $\mathbf{h}$ is the $1 \mathrm{x} n_{t}$ channel matrix given by ( 8$)$.

And

$$
\mathbf{h}=\left[h_{1}, h_{2}, \ldots \ldots, h_{n t}\right]
$$

$$
\mathbf{x}=\left[x_{1}, x_{2}, \ldots \ldots, x_{n t}\right]^{T}
$$

Where the elements $h i, i=1,2, \ldots \ldots, n_{t}$, represent the constant gain of the channel between the $i_{\text {th }}$ transmitter antenna and the single receiver antenna over a symbol period and $n_{t}$ is the transmitted antennas. The channel capacity of the discrete-time MISO channel model is represented by (10)[9].

$$
\mathrm{C}=\log _{2}\left(1+\frac{\rho}{n t} \sum_{i=1}^{n t}|h i|^{2}\right) b / s / H z
$$

\subsection{Mimo CAPACITY}

MIMO system offers a significant capacity gain over a traditional SISO channel, given that the underlying channel is rich of scatters with independent spatial fading. MIMO systems offered un increasing in channel capacity based on the utilization of space (or antenna) diversity at both the transmitter and the receiver and also on the number of antennas at both sides. A flat fading communications link was considered in this paper with single user, where the transmitter and receiver are equipped with $n_{t}$ and $n_{r}$ antennas respectively as shown by Figure 5. The received signal is represented by (11).

$$
y(i)=H(i) x(i)+n(i)
$$

Where, $y(i)$ is the $n_{r} \times 1$ received signal vector and $x(i)$ is the $n_{t} \times 1$ transmitted signal vector. $H(i)$ in (11) is an $n_{r} \times n_{t}$ matrix with a complex fading coefficients and its elements assumed to having an independent and identically distributed (i.i.d) circularly symmetric complex Gaussian random variables with zero mean and a variance of $1 / 2$ per dimension which leading to a Rayleigh fading channel model [10].

Also, $E\left\{n(i) n(i)^{H}\right\}=N_{0} I n_{r}$, where $I n_{r}$ denotes the $n_{r} \times n_{t}$ identity matrix and $E\{$.$\} refer to$ statistical avarege.
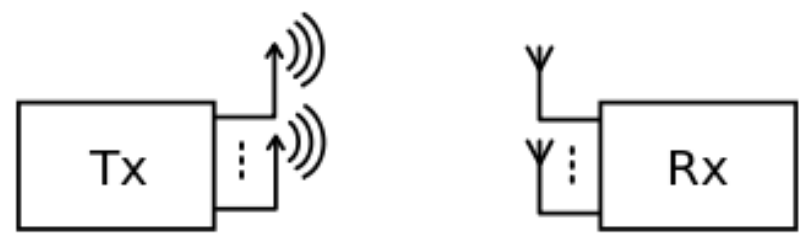

Figure 5. MIMO channel

MIMO system capacity in terms of spectral efficiency i.e. bits per second per $\mathrm{Hz}$, is represented by (12) [11].

$$
\mathrm{C}=\left[\log _{2} \operatorname{det}\left(\mathbf{I}_{\mathrm{nr}}+\frac{\mathrm{E}_{\mathrm{s}}}{\mathrm{n}_{\mathrm{t}} \mathrm{N}_{\mathrm{o}}} \mathrm{HQH}^{\mathrm{H}}\right)\right] b / s / \mathrm{Hz}
$$


Where, "det" means determinant, $\boldsymbol{I}_{n r}$ is the $n_{r} \times n_{t}$ identity matrix, $(\cdot)^{\mathrm{H}}$ means the Hermitian transpose (or transpose conjugate) and $\mathbf{Q}$ is a covariance matrix of the transmitter vector $\mathbf{x}$.

By considering a narrow-band single user MIMO system, the vector notation of the linear link model between the transmitter and receiver antennas can be represented by (13).

$$
y=\mathbf{H} x+\mathbf{N}
$$

Where $\mathrm{H}$ is the $n_{r} \times n_{t}$ normalized channel matrix, which can be represented as:

$$
\mathbf{H}=\left(\begin{array}{cccc}
h_{11} & h_{12} & \ldots & h_{1 n t} \\
h_{21} & h_{22} & \ldots & h_{2 n t} \\
\vdots & \vdots & \vdots & \vdots \\
h_{n r 1} & h_{n r 2} & \ldots & h_{n r n t}
\end{array}\right)
$$

Each element $h_{n r n t}$ represents the complex gains between transmitter and the receiver antennas.

\section{Equal Power Allocation}

Without CSI information at the transmitter, but perfectly known to the receiver, the optimum power allocation is to divide the available transmit power equally among the antennas elements of the transmitter. The capacity in (12) can be reduces to a new formula given by (15), by assuming that the components of the transmitted vector $\mathrm{x}$ as statistically independent, meaning that $\mathrm{Q}=\mathrm{I}_{\mathrm{nt}}$ with Gaussian distribution.

$$
\mathrm{C}=\left[\log _{2} \operatorname{det}\left(\mathrm{I}_{\mathrm{nr}}+\frac{\mathrm{E}_{\mathrm{s}}}{\mathrm{n}_{\mathrm{r}} \mathrm{N}_{\mathrm{o}}} \mathrm{HH}^{\mathrm{H}}\right)\right]
$$

It is clear from (15) that the MIMO channel capacity grows linearly for a case of $n_{r}=n_{t}$ rather than logarithmically $[12,13,14]$.

By letting $\mathrm{HH}^{\mathrm{H}}=\mathrm{VDV}^{\mathrm{H}}$ (Eigen Value Decomposition theorem), (15) can be reduced to (16)

$$
C=\left[\log _{2} \operatorname{det}\left(I_{n r}+\frac{E_{s}}{n_{r} N_{o}} V D V^{H}\right)\right]
$$

Where, $E_{S} / N_{o}$ is signal to noise ratio, $V$ is an $n r x n r$, matrix (eigenvectors of the channel $\mathrm{H}$ ) satisfying $V^{H} V=V V^{H}=I_{n}$, and $D=\operatorname{diag}\left\{\lambda_{1}, \lambda_{2}, \ldots, \lambda_{i}\right\}$ with. $\lambda_{i}>0$. The MIMO channel capacity in (16) can be reduces to a new formula represented by (17), based on the fact that the eigen values $\left(\lambda_{i}\right)$ of the channel $\mathrm{H}$ was already comprised into the diagonal matrix $D$.

$$
C=\left[\sum_{i=1}^{r} \log _{2}\left(1+\frac{E_{s}}{n_{t} N_{o}} \lambda_{i}\right)\right]
$$

Where, $\mathrm{r}=\operatorname{rank}\left(\mathrm{HH}^{\mathrm{H}}\right)=\min \left[n_{r}, n_{t}\right]$ (number of parallel channels) and, $(i=1, \ldots, r)$ are the positive eigen values of $\mathrm{HH}^{\mathrm{H}}$.MIMO channel capacity Expressed in (17) represents the sum of the capacities of $r$ SISO channels, each having power gain, $(i=1, \ldots, r)$ and transmit power $E_{s} / n_{t}$. It is noticed that all Eigen channels are allocated the same power; this is because these eigen channels are not accessible due to the lack of knowledge in the transmitter, i.e. no CSI, so it just divides the power equally among them $[14,15]$. 


\section{Perfect Csi At The Transmitter}

When CSI technique is applied, the channel matrix $\mathbf{H}$ will be feedback to the transmitter from the receiver, so the transmitter will know the channel matrix here before it transmits the data vectors. As shown in Figure. 6, the downlink transmitter receives partial CSI feedback $f^{\prime}$ from the receiver.

The true channel matrix, which the transmitter does not fully know, can modeled as a Gaussian random matrix (or vector) who's mean and covariance is given in the feedback [16]. In this case of action , MIMO system capacity can be improved based on using water filling principle, through assigning different levels of transmit power to various transmitting antennas [17].

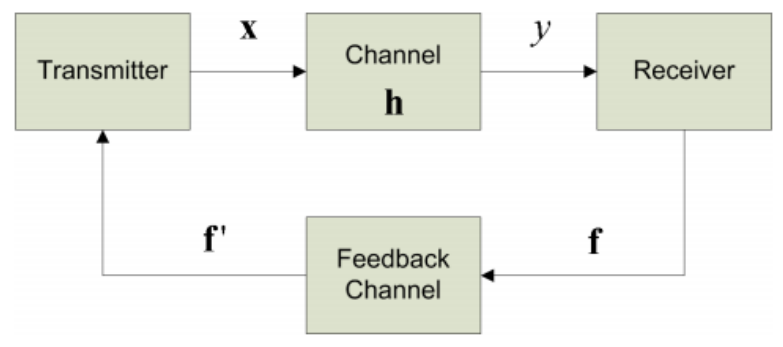

Figure 6. CSI system configuration

Water filling principle is an optimum solution related to a channel capacity improvement. It can be used to maximize the MIMO channel capacity through allocating of more power to the transmitted channels that are in good condition and less or none at all to the bad channels.

The capacity of an $\mathrm{n}_{\mathrm{r}} \times \mathrm{n}_{\mathrm{t}}$ MIMO channel with perfect CSI at the transmitter is represented by (18)[17].

$$
C_{C S I T}(H)=\underbrace{\max }_{Q \geq 0: \operatorname{Tr}\{Q\}=1} \log _{2} \operatorname{det}\left[\boldsymbol{I}_{n r}+\rho H \boldsymbol{Q} H^{H}\right]
$$

Where $\rho$ is a signal to noise ratio.

To obtain the optimum input covariance matrix $\left(\mathbf{Q}=\mathbf{Q}^{*}\right)$,the transmission is first decoupled along the individual channel modes, so that $n$ parallel data channels are formed in the directions of the singular vectors of the channel matrix $H$ at both the transmitter and the receiver. Along these

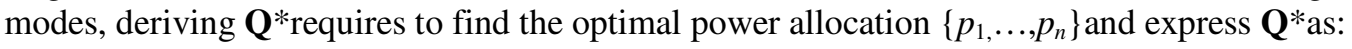

$$
\boldsymbol{Q}^{*}=V_{H} \operatorname{diag}\left\{P_{1}^{*}, \ldots, P_{n}^{*}\right\} V_{H}^{H}
$$

Here $\mathrm{V}_{\mathrm{H}}$ is given by the SVD of $\mathrm{H}$

$$
H=U_{H} \sum_{H} V_{H}^{H}
$$

and $\sum \mathrm{H}=\operatorname{diag}\left\{\sigma_{1}, \ldots, \sigma_{n}\right\}$, and $\sigma_{k}^{2} \triangleq \lambda_{K} \triangleq \lambda_{K}(W)$ with $\mathrm{W}=\mathrm{HH}^{H}$ (for $\left.n_{t}>n_{r}\right)$ or $\mathrm{W}=\mathrm{H}^{H} \mathrm{H}\left(\right.$ for $\left.n_{t}<n_{r}\right)$, then the MIMO channel capacity under perfect CSI, can be represented by (21).

$$
C_{C S I T}(H)=\underbrace{\max }_{\{P K\}_{K=1}^{n}} \sum_{k=1}^{n} \log _{2}\left[1+\rho P_{K} \lambda_{K}\right]
$$




$$
=\sum_{K=1}^{n} \log _{2}\left[1+\rho P_{K}^{*} \lambda_{K}\right]
$$

The optimum powers allocated $\operatorname{are}\left\{P_{1}^{*}, \ldots \ldots, P_{n}^{*}\right\}$, which resulting from applying the well-known water-filling algorithm.

\section{Correlated Mimo Channels}

The most fundamental technique to achieve diversity is to separate sufficiently more than one antenna from each other so that the relative phases of the multipath contributions are significantly different at the two adjacent antennas. When large phase differences are present between the total signals received at each of the antennas, this indicates that there is a low correlation between the signals at the antennas. Correlation factor decreases with increase in the distance between the antennas and this distance must be not less than $\lambda / 2$. More details concerning the effect of correlation on MIMO channel capacity can be found in reference [18]. Rayleigh correlated channel was considered here and was represented by (22), using Kronecker model[19].

$$
H_{w}=R_{r}^{\frac{1}{2}} H_{i i d} R_{t}^{\frac{1}{2}}
$$

Where $H_{w}$ is the correlated channel matrix, $R_{r}$ is the receive correlation matrix, $R_{t}$ is the transmit correlation matrix, and $H_{i i d}$ is the uncorrelated channel matrix. MIMO system capacity considering correlated channel is represented by (23).

$$
\mathrm{C}=\mathrm{E}_{\mathrm{H}}\left[\log _{2} \operatorname{det}\left(\mathrm{I}_{\mathrm{nr}}+\frac{\mathrm{E}_{\mathrm{s}}}{\mathrm{n}_{\mathrm{t}} \mathrm{N}_{\mathrm{o}}} \mathrm{H}_{\mathrm{w}} \mathrm{QH}_{\mathrm{w}}^{\mathrm{H}}\right)\right]
$$

\section{ERgodic CAPACity}

By taking the ensemble average of the information rate over the distribution of the elements of the channel matrix $\mathrm{H}$, we obtain the ergodic capacity of a MIMO channel. This capacity is represented by (24) [20].

$$
\bar{C}=E\{I\}
$$

Without CSI information at the transmitter, the MIMO ergodic capacity is represented by (25).

$$
\overline{\mathrm{C}}=E\left\{\log \left(\operatorname{det}\left(I+\frac{p}{n t} H H^{H}\right)\right)\right\}
$$

With the presents of CSI information at the transmitter, MIMO ergodic capacity is represented by (26).

$$
\bar{C}=E\left\{\sum_{K=1}^{n} \log _{2}\left[1+\rho P_{K}^{*} \lambda_{K}\right]\right\}
$$

Where, $\lambda_{K}$ is the singular values of matrix Hand $P_{k}^{*}$ is the power coefficient that corresponds to the amount of power assigned to the $k_{\mathrm{th}}$ sub-channel. This coefficient is represented by (27).

$$
P_{k}^{*}=E\left\{\left|s_{k}\right|^{2}\right\}
$$




\section{Simulation ANd Results}

MIMO channel capacity over Rayleigh fading channel was simulated using MATLAB software for different antenna configurations, different SNR, uncorrelated and correlated channel (different correlation coefficients), and with and without CSI. It is found difficult to derive the closed-form expression for MIMO capacity to plot these capacity versus SNR, therefore Monte Carlo simulation is used, i.e., a large number around (1000) of random matrices His generated, and then the average of capacity $(\bar{C})$ is taken, by using a software packege for this purpose.

\subsection{Uncorrelated and Equal Power}

Figure.7 shows the results for uncorrelated channel, where the system capacity is simulated for SIMO, MISO and MIMO as a function of antennas number at $5 \mathrm{~dB}$ SNR, for transmitter diversity MISO and receiver diversity SIMO, capacity leading to logarithmic growth (bounded).In MIMO system the capacity is linearly growing and much more considerable than MISO and SIMO systems.

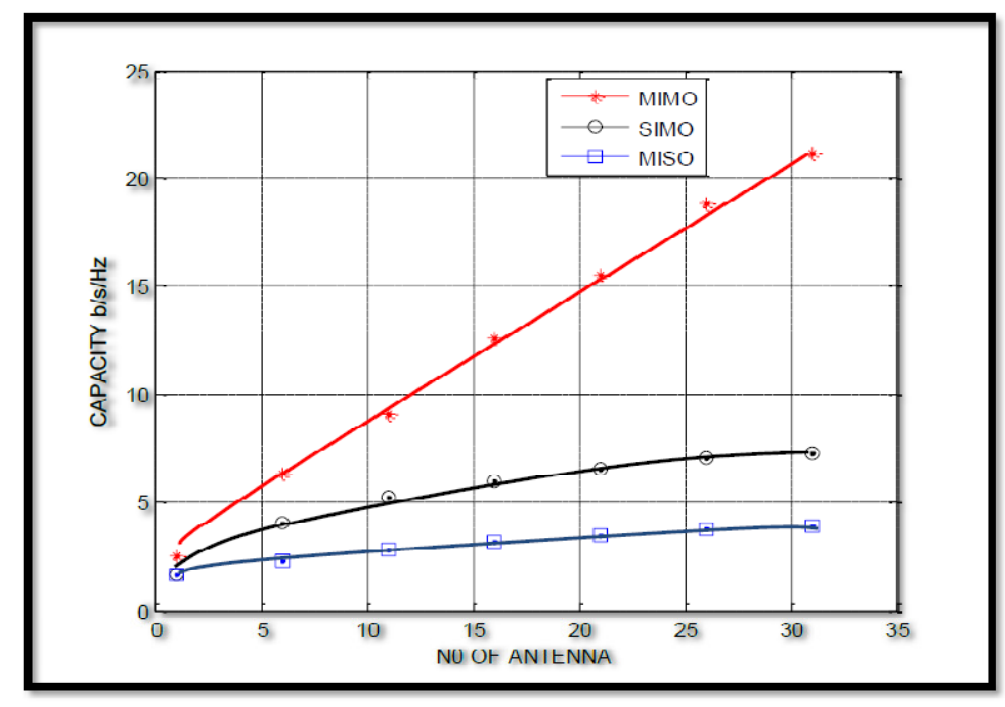

Figure.7 The capacity for MIMO, SIMO, and MISO structures

Figure. 8 depicts MIMO capacity for different antenna configurations as a function of SNR. It is clearly show that the capacity will increase with increasing SNR and also with the numbers of $\left(\mathrm{n}_{\mathrm{t}} \mathrm{x} \mathrm{n}_{\mathrm{r}}\right)$. 


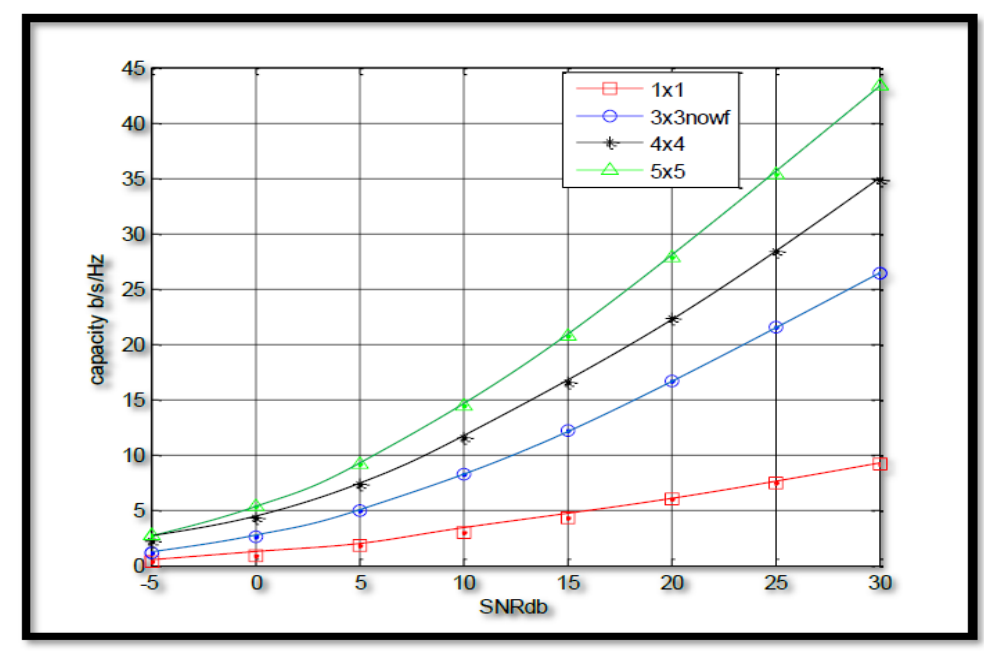

Figure 8.Capacity of different MIMO antenna configurations versus the SNR in $\mathrm{dB}$

Figure 9 show the MIMO capacity variation with $\left(n_{t} \times n_{r}\right)$ for different values of SNR. It can be seen that for $(5 \times 5)$ channel the capacities becomes 2, 9 and $19 \mathrm{bit} / \mathrm{s} / \mathrm{Hz}$ for SNR 0,10 and $20 \mathrm{~dB}$ respectively, while for $(8 \times 8)$ the capacities becomes 4,18 and 36 respectively ,i.e., multiply by factor of 2 .

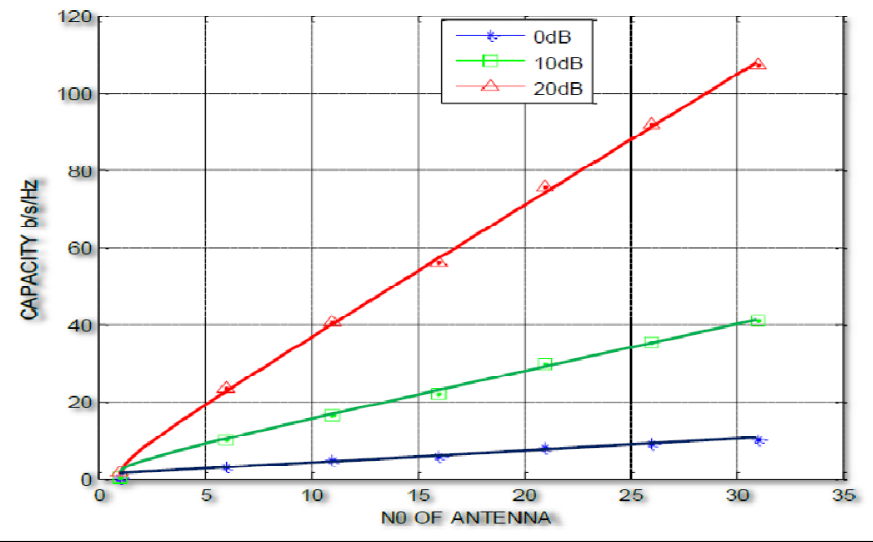

Figure 9.MIMO capacity for different SNR-dB versus number of antenna

\subsection{Uncorrelated Mimo Channel With Csi}

The effect of Channel State Information (CSI) at the transmitter on the overall channel capacity, with water filling (wf) algorithm to allocate the power among the transmitter antennas, was investigated here and shown in Figures 10 and 11.

To improvement the capacity by performing wf, is considerable for low $\mathrm{SNR}=3 \mathrm{~dB}$ and a large number of transmit or received antennas. Figure 10 show that by varying the number of the receiving antenna a limited gain in the capacity is obtained when fixing the number of the transmitting antenna. 


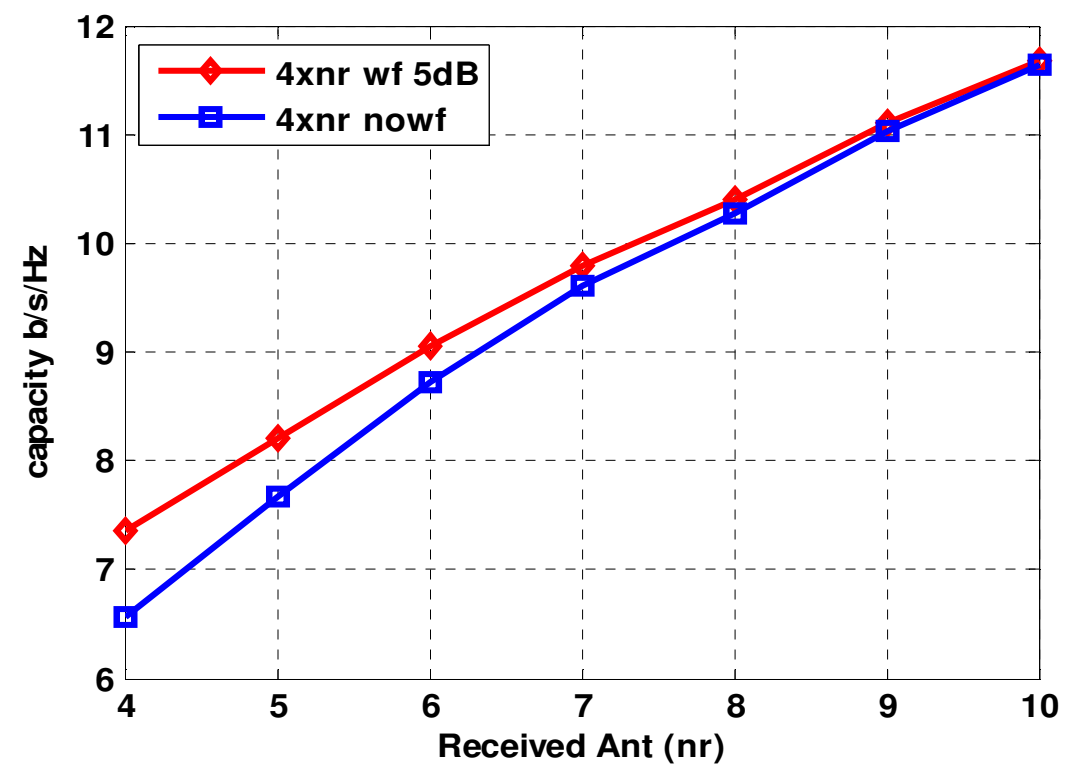

Figure 10.MIMO capacity with varying $n_{r}$ and $n_{t}=4, \mathrm{SNR}=3 \mathrm{~dB}$

Figure 11 show that by varying the number of the transmitting antenna a considerable gain in the system capacity is obtained when fixing the number of the receiving antenna.

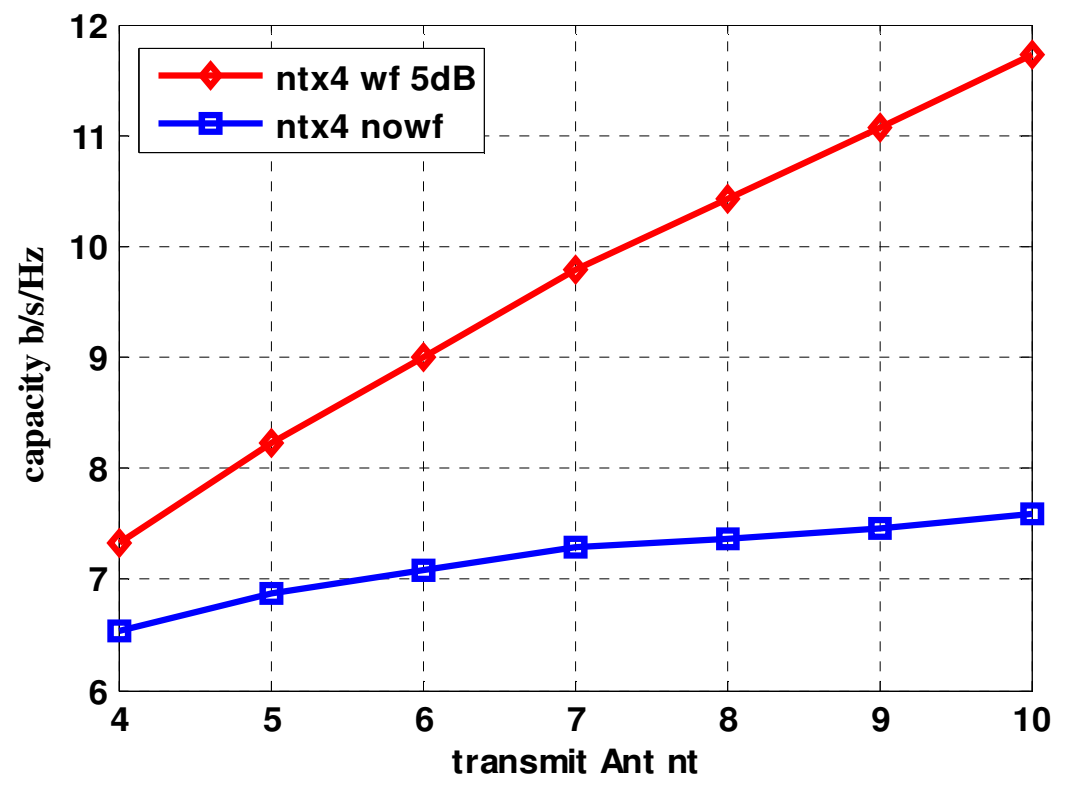

Figure 11.MIMO capacity with varying $n_{t}$ and $n_{r}=4, \mathrm{SNR}=3 \mathrm{~dB}$

Figure. 12 shows the variation of capacity with correlation coefficients(r) for $(4 \times 4)$ channel at $5 \mathrm{~dB}$ SNR. By decreasing the distance between antennas, correlation coefficient will increase, leading to decreasing in system capacity, but still we have considerable gain in case of CSI. 


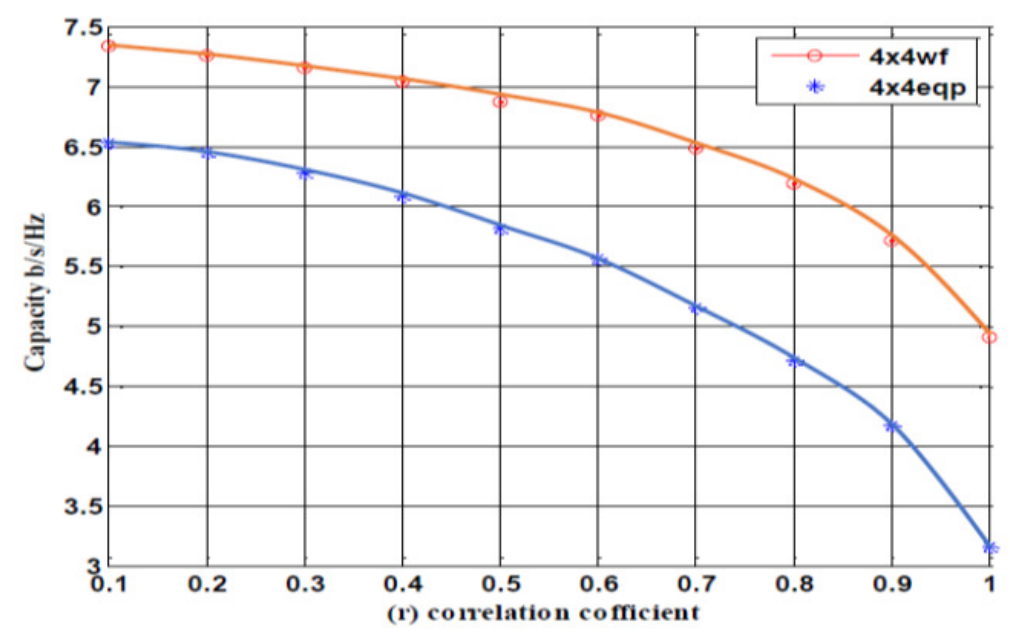

Figure 12. (4*4) MIMO channel capacity variation with correlation coefficients(r) at $\mathrm{SNR}=5 \mathrm{~dB}$

\section{CONCLUSION}

MIMO system capacity was investigated here for different system parameters using MATLAB software simulation. Simulation result shows that MIMO system has a considerable benefit in capacity compared with SIMO and MISO system. System capacity was considered for different MIMO system configuration at both transmitter and receiver by varying the number of antennas. Simulations results show that the capacity was improved when CSI information was known at the transmitter by applying Water Filling algorithm. By varying the number of the transmitting antenna a considerable gain in the system capacity is obtained when fixing the number of the receiving antenna. Finally, results shows that MIMO channel capacity will decrease when the correlated factor increase, but still we have a considerable gain in case when applying CSI technique.

\section{REFERENCES}

[1] R.Gray and D.Ornstein, "Block coding for discrete stationary -continuous noisy channels," Information Theory, IEEE Transactions on, vol. 25, no. 3, pp. 292 - 306, may 1979.

[2] I.E.Telatar, "Capacity of multi-antenna gaussian channels." tech. rep., AT \& TBell.

[3] P.Sunil Kumar, M.G.Sumithra, M.Sarumathi, " Performance Evaluation of Antenna Selection Techniques to Improve the Channel Capacity in MIMO Systems," Department of ECE, Bannari Amman Institute of technology, Tamil Nadu, India, July 2-3, 2013.

[4] J.H.Winters, B "Optimum combining in digital mobile radio with co-channel interference", IEEE J. Sel. Areas Commun, vol. SAC-2, no. 4, pp. 528-539, Jul.1984.

[5] C.N.Chuah, D. N.C.Tse, and J.M.Kahn, "Capacity scaling inMIMO wireless systems under correlated fading", IEEE Transactions inInformation Theory, vol.48, No. 3, pp. 637-659, March 2002.

[6] Shannon, C.E., "A Mathematical Theory of Communications," Bell Systems Technology Journal, Vol. 27, pp. 379-423, July 1948.

[7] Shannon, C.E., "A Mathematical Theory of Communications," Bell Systems Technology Journal, Vol. 27, pp. 623-656, October 1948.

[8] Shannon, C.E., "Communication in the Presence of Noise," Proceedings of the IEEE, Vol. 86, No. 2, pp. 447-457, February 1998 (this paper is reprinted from the proceedings of the IRE, Vol. 37, No. 1, pp. 10-21, January 1949).

[9] Gesbert, D., et al., "From Theory to Practice: An Overview of MIMO Space-Time Coded Wireless Systems," IEEE Journal on Selected Areas in Communications, Vol.21, No. 3, pp. 281-302, April 2003. 
[10] Laxminarayana S. Pillutla and Sudharman K. Jayaweera," Capacity of MIMO Systems in Rayleigh Fading with Sub-Optimal Adaptive Transmission Schemes" Department of Electrical and Computer Engineering Wichita State University. October 10-13, 2004

[11] Int. J.Communications, Network and System Sciences, 2010, 3, 213-252 doi:10.4236/ijcns.2010.33031 blished Online March 2010 (http://www.SciRP.org/journal/ijcns/).

[12] Telatar, I.E., "Capacity of Multi-antenna Gaussian Channels," European Transactionson Communications, Vol. 10, No. 6, pp. 585-595, 1999.

[13] Foschini, G.J., "Layered Space-Time Architecture for Wireless Communication in a Fading Environment When Using Multi-Element Antennas," Bell Labs TechnicalJournal, pp. 41-59, autumn 1996.

[14] Foschini, G.J., et al., "Analysis and Performance of Some Basic Space-Time Architectures," IEEE Journal on Selected Areas in Communications, Vol. 21, No.3,pp. 303-320, April 2003.

[15] XiwuLv, Kaihua Liu, Yongtao Ma, " Some results on the capacity of MIMO Rayleigh fading channels." School of Electronic and Information EngineeringTianjin UniversityTianjin, China.

[16] F. Khalid and J. Speidel, "Advances in MIMO Techniques for Mobile Communications-A Survey," Int'1 J. of Communications, Network and System Sciences, Vol. 3 No. 3, 2010, pp. 213-252.

[17] Claude Oestges and B Clerckx, "MIMO Wireless Communications: From Real World Propagation to Space-Time Code", Elsevier Ltd,pp110-115,2007.

[18] A. Paulraj, R.Nabar, and D.Gore.2003. Introduction to Space-Time Wireless Communications, Cambridge: Cambridge University Press, Chap. 4.

[19] A. Zelst and J.S. Hammerschmidt. "A single coefficient spatial correlation models for multiple-input multiple output (MIMO) radio channels", in Proc. URSI XXVIIth General Assembly, 2002.

[20] ArogyaswamiPaulraand RohitNabar‘DhananjayGor."Introduction to space time wireless communications", Cambridge university, pp.72-75, 2003.

\section{Author}

1. Eng. Emad. Mohamed was born in Mousel- Iraq on 1964.Lecturer atAlhdba University College-Mousel-Iraq. Received BSc degreefrom MTC College-BaghdadIraq in 1987.Received MSc degrees from EMU CyprusState University in 2013.Consultant in wireless communication.

2. Dr.Eng. A.M.Abdulsattar was born in Mousel-Iraq on 1954. Lecturer at AlhdbaUniversity College-Mousel-Iraq. R\&D contribution and consultant in wireless communication. Received BSc degrees from MTC College/Baghdad in 1977.

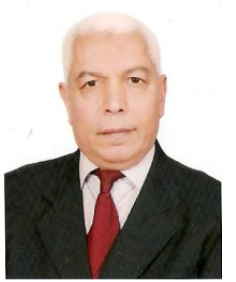

Received MSc and Ph.D degrees from ENSAE-France in 1979 and 1983 respectively. From 1983 to 1987 Lecturer at MTC College-Baghdad- Iraq. From 1987 to 2004 Researcher at Industrial sector for development of Electronic and Communication systems. From 2004 to 2006 contribute in development of a privet sector companies for wireless applications. From 2006 to 2014, Lecturer at Mousel University and Alhdba university college-Mousel-Iraq. Having many Publications in local and International magazines in the digital communication sector. 